\title{
Decision-Theoretic Paradoxes as Voting Paradoxes
}

\author{
Rachael Briggs \\ University of Sydney, Griffith University
}

It is a platitude among decision theorists that agents should choose their actions so as to maximize expected value. But exactly how to define expected value is contentious. Evidential decision theory (henceforth EDT), causal decision theory (henceforth CDT), and a theory proposed by Ralph Wedgwood that I will call benchmark theory (BT) all advise agents to maximize different types of expected value. Consequently, their verdicts sometimes conflict. In certain famous cases of conflict-medical Newcomb problems-CDT and BT seem to get things right, while EDT seems to get things wrong. In other cases of conflict, including some recent examples suggested by Egan 2007, EDT and BT seem to get things right, while CDT seems to get things wrong. In still other cases, EDT and CDT seem to get things right, while BT gets things wrong.

It's no accident, I claim, that all three decision theories are subject to counterexamples. Decision rules can be reinterpreted as voting rules, where the voters are the agent's possible future selves. The problematic examples have the structure of voting paradoxes. Just as voting paradoxes show that no voting rule can do everything we want, decision-theoretic paradoxes show that no decision rule can do everything we want. Luckily, the so-called "tickle defense" establishes that EDT, CDT, and BT will do everything we want in a wide range of situations. Most decision situations,

I thank Dan Greco, Caspar Hare, Brian Hedden, Vann McGee, and an anonymous reviewer at the Philosophical Review for their helpful comments. 
I argue, are analogues of voting situations in which the voters unanimously adopt the same set of preferences. In such situations, all plausible voting rules and all plausible decision rules agree.

\section{Background Assumptions}

The decision theories I'll discuss all rest on a common conceptual framework. All three theories advise agents about how to behave in decision situations. An agent's ultimate goal in a decision situation is to attain the outcome with the highest value. But since agents in decision situations are typically uncertain as to how much value their actions will engender, they typically do not know how to maximize value directly. The best they can do is to maximize expected value. The three theories I will discuss provide different conceptions of expected value.

Before I begin with a more detailed discussion of decision situations, a bit more discussion of value is in order. I will assume that values can be meaningfully represented using real numbers, so that (for example) an outcome with a value of thirty is better than an outcome with a value of twenty and worse than an outcome with a value of fifty, but twice as close (in some meaningful sense) to the former as to the latter. There needn't be anything special about either the value zero or the size of the units: all of the decision theories I discuss give the same results under positive linear transformations. (That is, where $a$ is any positive real number and $b$ is any real number, taking the value of each option, multiplying by $a$, and adding $b$ will not affect the decision theories' prescriptions.)

I will write ' $V=v$ ' for the proposition that the agent obtains an outcome with value $v$, and I will refer to propositions of the form ' $V=v$ ' as value-level propositions. I make absolutely no substantial assumptions about the nature of the good measured by the value function: 'value' may be read hedonically, morally, aesthetically, pragmatically, or in whatever other way suits the reader's fancy.

On to decision situations. I'll represent each decision situation as a triple $\langle\mathcal{A}, \mathcal{K}, C\rangle$, where $\mathcal{A}$ is a finite set of possible actions that the agent believes are available, $\mathcal{K}$ is a finite set of dependency hypotheses about how various value-level propositions depend counterfactually on the possible actions, and $C$ is a credence function representing the agent's beliefs. I'll elaborate on each member of the triple in turn.

First, the actions. By convention, the actions in $\mathcal{A}$ are pairwise incompatible: if the agent can walk, chew gum, or do both at the same time, then the set of actions will include walking and chewing gum and chewing gum and not walking, but it will not include walking or chewing gum. 
By convention, the actions in $\mathcal{A}$ are also jointly exhaustive: if the agent can refrain from both walking and chewing gum, then not walking and not chewing gum will count as an action (at least assuming that there is nothing else the agent can choose to do or not do at the same time). I will use the same symbol to denote both an action and the proposition to the effect that the action is performed; as far as I know, nothing important hinges on this ambiguity.

Next, the dependency hypotheses. These are sets of counterfactual conditionals. More precisely, each $K \in \mathcal{K}$ is a set containing, for every $A \in \mathcal{A}$, exactly one nonbacktracking counterfactual conditional of the form $A \square \rightarrow(V=v)$ and nothing else. ${ }^{1}$ This provides a new way to write the value function: I will write $V(K \& A)$ for the $v$ such that $(A \square \rightarrow(V=v)) \in K$.

I'll make two substantial assumptions about the nature of dependency hypotheses. First, I'll assume that the actions in $\mathcal{A}$ are causally irrelevant to the dependency hypotheses in $\mathcal{K}$-in other words, that an agent cannot change what depends on his or her actions by acting. Second, I'll assume that in every possible world, some dependency hypothesis $K$ is true-in other words, that no matter what, for every $A \in \mathcal{A}$, there is some amount of value $v$ that the agent would enjoy if he or she were to perform $A$. I will use the same symbol to denote both a given dependency hypothesis and a proposition to the effect that all the counterfactuals in that dependency hypothesis are true; as far as I know, nothing important hinges on this ambiguity.

The information encoded in $\mathcal{A}$ and $\mathcal{K}$ can be written in matrix form. Consider a simple situation in which an agent is at the racetrack, deciding whether to bet fifty dollars on horse 1 or on horse 2. Suppose horse 1 is running at three-to-one odds, while horse 2 is running at nine-toone odds. Assuming that the agent cares only about money, and assuming that he or she values money linearly, the available actions and dependency hypotheses can be written as follows (where betting on horse 1 is abbreviated ' $A_{1}$ ', and betting on horse 2 is abbreviated ' $A_{2}$ ').

\begin{tabular}{|l|l|l|l|}
\hline & $K_{1}$ & $K_{2}$ & $K_{3}$ \\
\hline$A_{1}$ & 150 & -50 & -50 \\
\hline$A_{2}$ & -50 & 450 & -50 \\
\hline
\end{tabular}

1. For the purposes of this essay, I assume that the counterfactual dependency between actions and value-level propositions is completely deterministic. See Lewis 1981 for a discussion of probabilistic counterfactual dependency in causal decision theory. 
The content of each dependency hypothesis can be read off from the corresponding column of the matrix. If the cell in column $K_{i}$ and row $A_{j}$ contains the value $v$, then $\left(A_{j} \square \rightarrow(V=v)\right) \in K_{i}$. So in the above matrix, $K_{1}$ is the set $\left\{A_{1} \square \rightarrow(V=150), A_{2} \square \rightarrow(V=-50)\right\}$-the dependency hypothesis that obtains if and only if horse 1 wins.

Finally, the credence function $C$. An agent facing a decision situation does not know what he or she will do and may not know which dependency hypothesis is true. However, the agent has partial beliefs about both members of $\mathcal{A}$ and members of $\mathcal{K}$. These are represented by the credence function $C$, which assigns real numbers between zero and one to arbitrary Boolean compounds of elements of $\mathcal{A}$ and $\mathcal{K}$. I'll assume that $C$ is a probability function satisfying the Kolmogorov axioms.

The information encoded in $C$ can be written in matrix form. The credence function of the agent in the racetrack situation, for instance, might be described as follows.

\begin{tabular}{|l|l|l|l|}
\hline & $K_{1}$ & $K_{2}$ & $K_{3}$ \\
\hline$A_{1}$ & .1 & .05 & .35 \\
\hline$A_{2}$ & .1 & .05 & .35 \\
\hline
\end{tabular}

Each cell in the matrix represents a conjunction, and the entry in a given cell represents the credence that the agent assigns to that conjunction. For instance, since the cell in column $K_{1}$ and row $A_{1}$ contains the entry .1 , the matrix says that $C\left(K_{1} \& A_{1}\right)=.1$. Since $C$ is a probability function, and since the conjunctions represented by the cells are pairwise incompatible and jointly exhaustive, the entries in the cells must add up to one.

So every decision situation $\langle\mathcal{A}, \mathcal{K}, C\rangle$ can be represented as a pair of matrices: the first representing $\mathcal{A}$ and $\mathcal{K}$, and the second representing $C$. Notice that on this method of representation $C(V=v)$ can be read off the two matrices jointly, though it cannot be read off either matrix individually. To compute $C(V=v)$, just examine the first matrix (representing $\mathcal{A}$ and $\mathcal{K}$ ), identify all the cells containing the entry $v$, and add up the entries of the corresponding cells in the second matrix.

One final note on credence functions: I will find it useful to speak of evidential independence. I will say that propositions $A$ and $B$ are evidentially independent (for an agent with a credence function $C$ ) just in case $C(A \mid B)=C(A)$. Evidential independence is always relative to the agent's credence function $C$, but I will leave this relativization implicit. 


\section{EDT}

EDT recommends that agents maximize evidentially expected value, or e-expected value for short. The e-expected value of an action $A \in \mathcal{A}$ is defined as follows.

$$
V_{E}(A)=\sum_{v} C(V=v \mid A) v
$$

In other words, $A$ 's e-expected value is a weighted average of the values of possible outcomes that might result from choosing $A$, where the weighting of each value $v$ is determined by the agent's conditional credence in the proposition $V=v$ given $A$. $V_{E}(A)$ measures the amount of value the agent would expect to enjoy, were he or she to conditionalize on the information that he or she had performed A. EDT can be summed up in the slogan: "When you have the power to make the news, make good news" (see Jeffrey 1983, 82-83).

One can give an alternative definition of e-expected value in terms of the dependency hypotheses.

$$
V_{E}(A)=\sum_{K \in \mathcal{K}} C(K \mid A) V(K \& A)
$$

This second definition is equivalent to the first and will later prove useful in explaining the relationship between CDT and EDT. ${ }^{2}$

2. Proof that the two definitions are equivalent: (2) can be rewritten as follows.

$$
V_{E}(A)=\sum_{v} \sum\{C(K \mid A): V(K \& A)=v\} v
$$

But for each $K \in \mathcal{K}$ such that $V(K \& A)=v, K \& A$ entails $V=v$. Furthermore if no $K \in \mathcal{K}$ such that $V(K \& A)=v$ is true, then some $K \in \mathcal{K}$ such that $V(K \& A) \neq v$ must be true. So for any $v$,

$$
\sum\{C(K \mid A): V(K \& A)=v\}=C(V=v \mid A) .
$$

Thus, we can rewrite (2) again as

$$
V_{E}(A)=\sum_{v} \sum\{C(V=v \mid A) v: V(K \& A)=v\} v
$$

Eliminating the inner sum,

$$
V_{E}(A)=\sum_{v} C(V=v \mid A) v
$$

But that's just (1). 
EDT faces counterexamples-so-called "medical Newcomb problems"-like the following (Egan 2007, 94):

\section{The Smoking Lesion}

Susan is debating whether or not to smoke. She believes that smoking is strongly correlated with lung cancer, but only because there is a common cause-a condition that tends to cause both smoking and cancer. Once we fix the presence or absence of this condition, there is no additional correlation between smoking and cancer. Susan prefers smoking without cancer to not smoking without cancer, and she prefers smoking with cancer to not smoking with cancer.

Evidently, Susan should smoke. Smoking dominates not smoking-Susan is better off smoking than not smoking no matter what. But EDT advises Susan not to smoke. Her smoking is bad news-it constitutes evidence that she will get cancer.

We can illustrate how EDT arrives at this verdict by modeling The Smoking Lesion using the matrices introduced in the previous section. Let $S$ be the proposition that Susan smokes. Whether Susan gets cancer or not depends on a host of additional unknown factors (none of which, I will assume, influence or are causally influenced by whether she smokes). Let $R$ be the proposition that these unknown factors conspire to ensure that Susan will get cancer. Then using the matrix formalism introduced in the previous section, we can represent Susan's actions and the dependency hypotheses in The Smoking Lesion as follows.

\begin{tabular}{|l|l|l|}
\hline & $R$ & $\neg R$ \\
\hline$S$ & -90 & 10 \\
\hline$\neg S$ & -100 & 0 \\
\hline
\end{tabular}

We can represent her credence function as follows.

\begin{tabular}{|l|c|c|}
\hline & $R$ & $\neg R$ \\
\hline$S$ & .37 & .13 \\
\hline$\neg S$ & .13 & .37 \\
\hline
\end{tabular}

By (1),

$$
\begin{aligned}
V_{E}(S) & =C(V=-90 \mid S)(-90)+C(V=10 \mid S)(10) \\
& =(.74)(-90)+(.26)(10) \\
& =-64
\end{aligned}
$$




$$
\begin{aligned}
V_{E}(\neg S) & =C(V=-100 \mid \neg S)(-100)+C(V=0 \mid \neg S)(0) \\
& =(.26)(-100)+(.74)(0) \\
& =-26 .
\end{aligned}
$$

$V_{E}(S)<V_{E}(\neg S)$, so EDT advises Susan not to smoke.

What's going on? Susan assigns a low value to any outcome where $R$ is true, and she thinks $R$ is much more likely given $S$ than it is given $\neg S$. This is not because she thinks $S$ has any propensity to cause $R$-on the contrary, she thinks $R$ and $S$ have a common cause (namely, the lesion). We need a decision theory that can distinguish Susan's beliefs about which outcomes her actions are liable to cause from her beliefs about which outcomes are merely correlated with her actions.

\section{CDT}

Several authors, including Gibbard and Harper (1978), Lewis (1981), Sobel (1994), and Joyce (1999), advocate CDT as a solution to medical Newcomb problems. CDT advises agents to maximize causally expected value, or c-expected value for short. ${ }^{3}$ The c-expected value of an action $A \in \mathcal{A}$ is defined as follows.

$$
V_{C}(A)=\sum_{v} C(A \square \rightarrow(V=v)) v
$$

In other words, $A$ 's c-expected value is a weighted average of the values of possible outcomes that might result from choosing $A$, where the weighting of each value $v$ is determined by the agent's credence in the proposition that if he or she were to perform $A$, some outcome with value $v$ would result. CDT can be summed up in the slogan: "Choose whichever action is likely to have the best results (according to your beliefs just before acting)."

3. An anonymous reviewer for the Philosophical Review points out that the names "causal decision theory" and "causally expected value" are somewhat misleading since the theory is stated in terms of counterfactuals rather than causation. "Counterfactual decision theory" and "counterfactually expected value" would be more apt, but I will continue to write "causal" rather than "counterfactual" in accordance with established usage. 
An equivalent definition of c-expected value can be given in terms of dependency hypotheses. ${ }^{4}$

$$
V_{C}(A)=\sum_{K \in \mathcal{K}} C(K) V(K \& A)
$$

Just as (2) expressed $A$ 's e-expected value as a weighted sum of terms of the form $V(K \& A)$, (4) expresses $A$ 's c-expected value as a weighted sum of terms of the form $V(K \& A)$. Although the terms are the same, the weightings are different. In the case of e-expected value, they are the agent's conditional credences in the $K$ s given $A$, while in the case of c-expected value, they are the agent's unconditional credences in the $K$ s.

Unlike EDT, CDT yields the right answer in The Smoking Lesion. By (4),

$$
\begin{aligned}
V_{C}(S) & =C(R) V(R \& S)+C(\neg R) V(\neg R \& S) \\
& =(.5)(-90)+(.5)(10) \\
& =-40 \\
V_{C}(\neg S) & =C(R) V(R \& \neg S)+C(\neg R) V(\neg R \& \neg S) \\
& =(.5)(-100)+(.5)(0) \\
& =-50 .
\end{aligned}
$$

$V_{C}(S)>V_{C}(\neg S)$, so CDT advises Susan to smoke.

Although EDT and CDT conflict in The Smoking Lesion, equations (2) and (4) show that they coincide whenever $C(K)=C(K \mid A)$ for each $K \in \mathcal{K}$ and $A \in \mathcal{A}$-that is, whenever the agent's actions are evidentially

4. Proof that the two definitions are equivalent: (2) can be rewritten as follows.

$$
V_{C}(A)=\sum_{v} \sum\{C(K):(A \square \rightarrow(V=v)) \in K\} v
$$

For any $v$ and any $K \in \mathcal{K}$ such that $(A \square \rightarrow(V=v)) \in K$,

$$
C(K) v=C(K) V(K \& A) .
$$

Thus, (2) can be rewritten again as follows.

$$
V_{C}(A)=\sum_{v} \sum\{C(K) V(K \& A):(A \square \rightarrow(V=v)) \in K\}
$$

For every $K \in \mathcal{K}$, there is exactly one $v$ such that $(A \square \rightarrow(V=v)) \in K$. So summing over the $v$ s is redundant. Thus,

$$
V_{C}(A)=\sum_{K \in \mathcal{K}} C(K) V(K \& A)
$$

But that's just (4). 
independent of the dependency hypotheses. Under ordinary circumstances, people consider their actions irrelevant to claims about what depends on their actions, so under ordinary circumstances, EDT and CDT coincide. In The Smoking Lesion, where the two theories diverge, CDT gets things right. One might be tempted to conclude that EDT is merely a good approximation to the truth, while CDT is exactly true. But several authors, Eells (1985), Bostrom (2001), Egan (2007), have formulated examples where EDT gets things right, while CDT gets things wrong.

I'll consider the following example from Egan 2007, 97; the other examples are similar in all relevant structural respects:

\section{The Psychopath Button}

Paul is debating whether to press the "kill all psychopaths" button. It would, he thinks, be much better to live in a world with no psychopaths. Unfortunately, Paul is quite confident that only a psychopath would press such a button. Paul very strongly prefers living in a world with psychopaths to dying.

Evidently, Paul should not press the button. If he presses, then by his own lights, he is very likely to cause his own death. But CDT advises him to press, at least if he starts out relatively confident in his own sanity.

We can illustrate how CDT arrives at this conclusion by using matrices to model The Psychopath Button. We can represent Paul's available actions and the dependency hypotheses as follows, where $B$ is the proposition that Paul pushes the button and $P$ is the proposition that Paul is a psychopath.

\begin{tabular}{|l|l|l|}
\hline & $P$ & $\neg P$ \\
\hline$B$ & -100 & 20 \\
\hline$\neg B$ & 0 & 0 \\
\hline
\end{tabular}

And we can represent his credence function as follows.

\begin{tabular}{|l|l|l|}
\hline & $P$ & $\neg P$ \\
\hline$B$ & .09 & .01 \\
\hline$\neg B$ & .01 & .89 \\
\hline
\end{tabular}

By (4),

$$
\begin{aligned}
V_{C}(B) & =C(P) V(P \& B)+C(\neg P) V(\neg P \& B) \\
& =(.1)(-100)+(.9)(20) \\
& =8
\end{aligned}
$$




$$
\begin{aligned}
V_{C}(\neg B) & =C(P) V(P \& \neg B)+C(\neg P) V(\neg P \& \neg B) \\
& =(.1)(0)+(.9)(0) \\
& =0 .
\end{aligned}
$$

$V_{C}(B)>V_{C}(\neg B)$, so CDT advises Paul to press-the wrong answer.

On the other hand, EDT advises him not to press-the right answer.

$$
\begin{aligned}
V_{E}(B) & =C(V=-100 \mid B)(-100)+C(V=20 \mid B)(20) \\
& =(.9)(-100)+(.1)(20) \\
& =-88 \\
V_{E}(\neg B) & =C(V=0 \mid \neg B)(0) \\
& =(1)(0) \\
& =0 \\
V_{C}(B)>V_{C}(\neg B) &
\end{aligned}
$$

What's going on? Paul's decision provides evidence about which dependency hypothesis is true. Before making his decision, he is confident that if he were to press the button, good things would happen. But conditional on his pressing the button, he is confident that if he were to press the button, bad things would happen. CDT effectively tells him to ignore the evidence that he would get by pressing the button.

EDT tells Paul to attend to the evidence he would get by pressing the button. According to EDT, Paul should perform the action that (by his current lights) will count as the strongest evidence that good things are about to happen. In The Psychopath Button, good things will happen if and only if Paul causes them-his actions aren't liable to give him evidence about good events on which they have no causal impact. Since EDT can account for the evidential impact of Paul's choice, and since there are no spurious correlations to lead it astray, it delivers the right result.

\section{BT}

What we need, it seems, is a theory that is sensitive to the agent's beliefs about the counterfactual consequences of the available actions (like CDT) but also sensitive to the evidence that the agent's actions provide about the causal structure of the world (like EDT). If our theory is to display appropriate sensitivity to the causal structure of the world, it should compare 
the values of actions within dependency hypotheses but should ignore any value differences between dependency hypotheses. This is because agents have complete control over the c-expected values of their actions within each dependency hypothesis but no control over which dependency hypothesis is true.

If our theory is to display appropriate sensitivity to the evidence provided by the agent's actions, it should consider the conditional comparative values of an agent's actions, given various hypotheses about which action he or she performs. The agent wants to choose an action which is the right one not just unconditionally but conditional on the proposition that he or she really performs it.

In an unpublished draft, Ralph Wedgwood (2009) suggests a way of developing the sort of decision theory at which I've just gestured. Within any dependency hypothesis $K \in \mathcal{K}$, Wedgwood suggests, there is a "benchmark" value-roughly, the default level of value to be gotten, given $K$. An action's causal goodness or badness can then be measured according to how far it exceeds or falls short of the benchmark. Thus, where $B_{K}$ is the benchmark value associated with $K$, the comparative value of $A$ within $K$ is

$$
C V_{K}(A)=V(K \& A)-B_{K} .
$$

Agents will typically be unsure about which $K \in \mathcal{K}$ is true, so they will typically be unable to calculate the comparative values of their actions directly. Still, they will be able to calculate expected comparative values for their actions. According to Wedgwood, it's appropriate for agents to use e-expectations rather than c-expectations. Both the e-expected comparative value of an action $A$ and the c-expected comparative value of an action $A$ are weighted averages of terms of the form $C V_{K}(A)$ for each $K \in \mathcal{K}$. In the case of e-expected comparative value, the weightings are the agent's conditional credences in the $K$ s given $A$, while in the case of c-expected comparative value, they are the agent's unconditional credences in the $K$ s. Wedgwood claims that it's best to use e-expected comparative values, weighted by the agent's conditional credences in the $K$ s given $A$, because the right action is the one that has the most advantage over the alternatives on the assumption that it is performed. Thus, agents should maximize

$$
V_{B T}(A)=\sum_{K \in \mathcal{K}} C(K \mid A)\left(V(K \& A)-B_{K}\right) .
$$


Wedgwood's theory might be called benchmark theory, or BT for short. Likewise, the sort of value measured by $V_{B T}$ might be called "bexpected value."

$A$ 's b-expected value as measured in (5), like $A$ 's c-expected value as measured in (2), is a weighted average of some quantity associated with the dependency hypotheses. In both (2) and (5), the weightings are determined by the agent's conditional credences in each of the $K$ s given $A$. But the quantities being averaged are different. In (2), the relevant quantity is simply the absolute value of $K \& A$. In (5), on the other hand, the relevant quantity is the relative value of $K \& A$ as compared to the default amount of value available in $K$.

So if EDT advises agents to maximize e-expected absolute value, $\mathrm{BT}$ advises them to maximize e-expected relative value-something like relief, the absence of regret. Thus, BT can be summed up in the slogan "Choose the action you expect to regret least."

An important question remains: how should the benchmark $B_{K}$ be determined for a given dependency hypothesis $K$ ? In an extreme vein, one might set $B_{K}$ equal to the value of the best outcome available in $K$. Taking the opposite extreme, one might set $B_{K}$ equal to the value of the worst outcome available in $K .^{5}$ Wedgwood rejects both of these extremes in favor of a more moderate option: first, eliminate all the actions that should not be taken seriously in practical reasoning (for example, actions that are dominated, or that the agent is certain won't be performed anyway) and second, take the average of the expected values of the remaining actions.

Notice that the first step, eliminating undesirable actions, is crucial. Wedgwood $(2009,23)$ writes,

In every situation in which we have to make a choice, there is an enormous number of perfectly dreadful courses of action that are at least physically (if not psychologically) "available." For example, you could commit a serious crime in a way that is certain to result in your being arrested; you could give every penny that you have to the politician whom you most despise; you

5. Loomes and Sugden (1982) call the quantity measured by the first option "regret" and the quantity measured by the second option "rejoice." My use of the word "regret" in the body of the essay is used in a looser sense-I merely intend to designate falling short of a benchmark, whether or not that benchmark is not set by the best possible action in the circumstances. Wedgwood uses "relief" for the quantity Loomes and Sugden call "rejoice"; again, my use of the word is intended in a looser sense-I merely intend to designate meeting or exceeding a benchmark, whether or not that benchmark is set by the worst possible action in the circumstances. 
could commit suicide in various agonizing ways, using various items that you keep in the cupboards and drawers of your kitchen; and so on. . . It does not seem that the bare fact that these courses of action are available should make a difference to what it is rational to choose.

In order to prevent these "insane" courses of action from affecting the benchmark, we must ignore them at the outset. ${ }^{6}$ Henceforth, I will assume that $\mathcal{A}$ is chosen to include only noninsane actions. This should not make a difference to the recommendations of EDT or CDT since I take it that if either EDT or CDT recommends an action, this is an adequate reason for its inclusion in the set of sane options.

So where $\# \mathcal{A}$ is the number of actions in $\mathcal{A}$ (once all insane actions have been excluded), Wedgwood holds that

$$
B_{K}=\frac{\sum_{A \in \mathcal{A}} V(K \& A)}{\# \mathcal{A}} .
$$

Recall that whenever the agent's actions are evidentially independent of the dependency hypotheses, EDT and CDT coincide. Under such circumstances, BT coincides with EDT and $\mathrm{CDT}^{7}$ In cases where EDT and CDT come apart, however, BT coincides sometimes with EDT and sometimes with CDT. In The Smoking Lesion, BT agrees with CDT. Using

6. Wedgwood goes on to suggest a second, dynamic way of setting a benchmark, but I will leave it aside. To the best of my knowledge, the complications it introduces do not substantially affect the point at hand.

7. Proof:

Suppose $C(K \mid A)=C(K)$ for every $K \in \mathcal{K}$ and $A \in \mathcal{A}$. Then for any $A \in \mathcal{A}$,

$$
\begin{aligned}
V_{B T}(A) & =\sum_{K \in \mathcal{K}}\left(V_{E}(K \& A)-B_{K}\right) C(K \mid A) \\
& =\sum_{K \in \mathcal{K}}\left(V_{E}(K \& A)-B_{K}\right) C(K) \\
& =\sum_{K \in \mathcal{K}} V_{E}(K \& A) C(K)-\sum_{K \in \mathcal{K}} B_{K} C(K) \\
& =V_{C}(A)-\sum_{K \in \mathcal{K}} B_{K} C(K) .
\end{aligned}
$$

Since the second term $\sum_{K \in \mathcal{K}} B_{K} C(K)$ is not a function of $A, V_{B T}(A)$ is maximized when $V_{C}(A)$ is maximized. Therefore, BT and CDT agree. Furthermore, BT and EDT agree under these circumstances, as I pointed out in the previous section. 
Wedgwood's method of setting benchmarks, the following equations hold.

$$
\begin{gathered}
B_{R}=\frac{(-90)+(-100)}{2}=-95 \\
B_{\neg R}=\frac{10+0}{2}=5
\end{gathered}
$$

Then, by (5),

$$
\begin{aligned}
V_{B T}(S)= & \left(V(R \& S)-B_{R}\right) C(R \mid S)+\left(V(\neg R \& S)-B_{R}\right) C(\neg R \mid S) \\
= & ((-90)-(-95))(.74)+(10-5)(.26) \\
= & 5 \\
V_{B T}(\neg S)= & \left(V(R \& \neg S)-B_{R}\right) C(R \mid \neg S) \\
& +\left(V(\neg R \& \neg S)-B_{R}\right) C(\neg R \mid \neg S) \\
= & ((-100)-(-95))(.74)+(0-5)(.26) \\
= & -5 .
\end{aligned}
$$

$V_{B T}(S)>V_{B T}(\neg S)$, so BT recommends $S$ - the intuitively right answer.

In The Psychopath Button, BT agrees with EDT. Using Wedgwood's method of setting benchmarks, the following equations hold.

$$
\begin{gathered}
B_{P}=\frac{-100+0}{2}=-50 \\
B_{\neg P}=\frac{20+0}{2}=10
\end{gathered}
$$

$$
\begin{aligned}
V_{B T}(B) & =\left(V(P \& B)-B_{P}\right) C(P \mid B)+\left(V(\neg P \& B)-B_{\neg P}\right) C(\neg P \mid B) \\
& =(-100-(-50)) .9+(20-10) .1 \\
& =-44
\end{aligned}
$$

$$
\begin{aligned}
V_{B T}(\neg B)= & \left(V(P \& \neg B)-B_{P}\right) C(P \mid \neg B) \\
& +\left(V(\neg P \& \neg B)-B_{\neg P}\right) C(\neg P \mid \neg B) \\
= & (0-(-50)) .1+(0-10) .9 \\
= & -4
\end{aligned}
$$

$V_{B T}(B)<V_{B T}(\neg B)$, so BT recommends $\neg B$-again, the intuitively right answer. 
So far, so good. Unfortunately, there's a serious problem with BT. ${ }^{8}$ The problem can be illustrated using an example cited by Egan (2007, 112) and attributed to Anil Gupta called The Three-Option Smoking Lesion. I have modified Egan's version of the example slightly to drive home just how severe the problem is.

\section{The Three-Option Smoking Lesion}

Samantha is deciding whether to smoke. But her situation is slightly more complicated than Susan's. Samantha has three options: Smoke cigars, smoke cigarettes, or refrain from smoking altogether... Due to the ways that various lesions tend to be distributed, it turns out that cigar smokers tend to be worse off than they would be if they were smoking cigarettes but better off than they would be if they refrained from smoking altogether. Similarly, cigarette smokers tend to be worse off than they would be smoking cigars but better off than they would be refraining from smoking altogether.

Finally, nonsmokers tend to be badly off no matter what they do. Smoking makes them neither better off nor worse off-the small gain in pleasure is exactly counterbalanced by the monetary cost.

Whatever Samantha does, she ought to smoke. Not smoking is weakly dominated - avoiding tobacco won't make Samantha better off and may make her worse off. Furthermore, not smoking has low e-expected value-smokers tend to be well off on average, while nonsmokers tend to be poorly off on average. But BT tells Samantha not to smoke.

Let Samantha's possible actions be labeled $A_{N}$ (not smoking), $A_{R}$ (smoking cigars), and $A_{T}$ (smoking cigarettes). Let the dependency hypotheses be labeled $K_{N}$ (Samantha has the kind of lesion that typically makes people nonsmokers), $K_{R}$ (Samantha has the kind of lesion that typically makes people smoke cigars), and $K_{T}$ (Samantha has the kind of lesion that typically makes people smoke cigarettes). These actions and dependency hypotheses might be characterized by the following matrix.

\begin{tabular}{|l|l|l|l|}
\hline & $K_{N}$ & $K_{R}$ & $K_{T}$ \\
\hline$A_{N}$ & 0 & 0 & 0 \\
\hline$A_{R}$ & 0 & 30 & 90 \\
\hline$A_{T}$ & 0 & 90 & 30 \\
\hline
\end{tabular}

8. I thank Caspar Hare for driving home to me the severity of the problem. 
Samantha's credence function might be characterized by the following matrix.

\begin{tabular}{|c|c|c|c|}
\hline & $K_{N}$ & $K_{R}$ & $K_{T}$ \\
\hline$A_{N}$ & .32 & .01 & .01 \\
\hline$A_{R}$ & .01 & .31 & .01 \\
\hline$A_{T}$ & .01 & .01 & .31 \\
\hline
\end{tabular}

Using Wedgwood's method of setting benchmarks, we can calculate $B_{N}$, $B_{R}$, and $B_{T}$.

$$
\begin{aligned}
& B_{N}=\frac{0+0+0}{3}=0 \\
& B_{R}=\frac{0+30+90}{3}=40 \\
& B_{T}=\frac{0+90+30}{3}=40
\end{aligned}
$$

$$
\begin{aligned}
V_{B T}\left(A_{N}\right)= & C\left(K_{N} \mid A_{N}\right)\left(V\left(A_{N} \& K_{N}\right)-B_{N}\right) \\
& +C\left(K_{R} \mid A_{N}\right)\left(V\left(A_{N} \& K_{R}\right)-B_{R}\right) \\
& +C\left(K_{T} \mid A_{N}\right)\left(V\left(A_{N} \& K_{T}\right)-B_{T}\right) \\
\approx & .9412(0-0)+.0294(0-40)+.0294(0-40) \\
\approx & -2
\end{aligned}
$$

$$
\begin{aligned}
V_{B T}\left(A_{R}\right)= & C\left(K_{N} \mid A_{R}\right)\left(V\left(A_{R} \& K_{N}\right)-B_{N}\right) \\
& +C\left(K_{R} \mid A_{R}\right)\left(V\left(A_{R} \& K_{R}\right)-B_{R}\right) \\
& +C\left(K_{T} \mid A_{R}\right)\left(V\left(A_{R} \& K_{T}\right)-B_{T}\right) \\
\approx & .0303(0-0)+.9394(30-40)+.0303(90-40) \\
\approx & -9 \\
V_{B T}\left(A_{T}\right)= & C\left(K_{N} \mid A_{T}\right)\left(V\left(A_{T} \& K_{N}\right)-B_{N}\right) \\
& +C\left(K_{R} \mid A_{T}\right)\left(V\left(A_{T} \& K_{R}\right)-B_{R}\right) \\
& +C\left(K_{T} \mid A_{T}\right)\left(V\left(A_{T} \& K_{T}\right)-B_{T}\right) \\
\approx & .0303(0-0)+.0303(30-40)+.9394(30-40) \\
\approx & -9
\end{aligned}
$$


Since $V_{B T}\left(A_{N}\right)$ is greater than both $V_{B T}\left(A_{R}\right)$ and $V_{B T}\left(A_{T}\right)$, BT advises Samantha not to smoke.

Wedgwood's response to this problem is to claim that since $A_{N}$ is weakly dominated, it is insane, and should be excluded from $\mathcal{A}$ at the outset (Wedgwood 2009, 38n). Although this response gets around the letter of the objection, it does not get around the the objection's spirit. Suppose Samantha stands to gain a tiny, tiny payoff if she refrains from smoking, provided (and only provided) she turns out to be a nonsmoker. Let the payoff be as tiny as you like-as tiny as a jelly bean, or a box top worth $1 / 200$ th of a cent. As long as the payoff is nonzero, refraining from smoking is not weakly dominated. Since it is not weakly dominated, Samantha has no reason to ignore it at the outset, and BT will tell her to choose it. Not smoking can be as close to weakly dominated as you like, and BT will still recommend it. Throwing out the weakly dominated options is ad hoc and does not address the real source of the problem.

\section{Decision Situations as Elections}

At this point, it's worth taking a step back to meditate on the situation. I suggest, as a useful mantra, Jeffrey's $(1983,16)$ slogan "Choose for the person you expect to become once you have chosen." Jeffrey used the slogan to advertise a particular modified version of EDT, but I wish to focus on a more general, less controversial interpretation of the idea.

Any choice you make is, in a broad sense, aimed at improving the welfare of the person you will become. Insofar as a decision of yours is justifiable, it ought to be justifiable to your future self-the person you will be immediately after acting. Insofar as possible, you should avoid doing things that your future self will deem ill advised.

EDT, CDT, and BT are all ways of choosing for the person you expect to become in this broad sense. EDT advises you to choose so that the person you expect to become is reasonably convinced that things will go well-whether or not there is any counterfactual link between your action and things going well. Your way of affecting your future self's beliefs is to cause him or her to form true beliefs about your actions, so convincing your future self that things will go well amounts to choosing the action that will make the best news. We might rephrase the slogan for EDT as follows: "Give your future self some good news!"

CDT advises you to choose so that the person you expect to become does better (by your current lights) than he or she would have done by 
choosing any other alternative. CDT might be understood as a kind of paternalism about your future self: it tells you to do what you think is counterfactually best for your future self. As The Psychopath Button illustrates, the action you think is counterfactually best for your future self may not constitute the best news from his or her perspective, and as The ThreeOption Smoking Lesion illustrates, your future self may not agree with you about what is counterfactually best. We might rephrase the slogan for CDT as follows: "Choose whichever action is likely to be best counterfactually for your future self (according to your current beliefs)."

BT advises a somewhat hybrid strategy. According to BT, you should choose so that the person you expect to become gets good news not about the absolute level of happiness that he or she is in for, but about the relative level of happiness that he or she is in for compared to other counterfactual selves who might have existed. BT advises you to choose so that (insofar as possible) your future self is reasonably convinced that his or her relative level of happiness is high. The parenthetical "insofar as possible" is needed because in decision situations like The Psychopath Button, no matter what you do, your future self will think her relative level of happiness is somewhat low. We might rephrase the slogan for BT as follows: "Insofar as possible, choose whichever action your future self is likely to think counterfactually best."

So Jeffrey's slogan is appealing in general, and not just in the narrow sense Jeffrey intended. Still, there is one respect in which the slogan can be improved. Talk of "the person you expect to become after choosing" is a little misleading since what you believe and desire later depend partly on what you choose. Speaking loosely, we might say that there is a collection of possible people you might become-one for each action you might choose. Better, then, to rephrase our mantra as follows: "Choose for the various possible people you might become."

Extending this metaphor further, we can envisage rational decision making as a democratic procedure in which you "poll" your possible future selves about their opinions regarding various available actions and then decide what to do based on their "answers." Which future self is actualized will depend on which action you end up performing. So your possible future selves satisfy two roles at once: they function as voters and as candidates for office.

The voting metaphor might seem a bit odd since you can't literally ask all your possible future selves what they think of your predicament. But there is a way of finding out what your possible future selves think. Let 
us assume that each possible future self has conditionalized, for a different $A \in \mathcal{A}$, on the proposition that you performed $A$. Let us also assume that your value function will remain the same before and after you choose. Then where $S^{A}$ is the self who will be actualized (or "elected to office") if you perform action $A, S^{A}$ 's credence function is $C^{A}=C(\cdot \mid A)$, and $S^{A}$ 's value function is simply $V$. By (4), the c-expected value that $S^{A}$ assigns to an action $B$ is

$$
V_{C}^{A}(B)=\sum_{K \in \mathcal{K}} C(K \mid A) V(B \& K)
$$

It's less clear how to calculate the e-expected values your possible future selves assign to the actions in $\mathcal{A}$. By (2), the e-expected value that $S^{A}$ assigns to an action $B$ should be

$$
V_{E}^{A}(B)=\sum_{K \in \mathcal{K}} C(K \mid A \& B) V(B \& K) .
$$

But members of $\mathcal{A}$ are pairwise incompatible, so for any distinct $A \in \mathcal{A}$ and $B \in \mathcal{A}, C(A \& B)=0$. Where $A \neq B$, it's not clear that $C(K \mid A \& B)$ is even well defined for any of the $K \in \mathcal{K}$. We might extend the definition of conditional probability in order to make $C(K \mid A \& B)$ well defined, but this would require us to add something to the information specified in the original decision situation. In the interests of simplicity and economy, then, it is best to extend the polling metaphor in terms of the c-expected values rather than the e-expected values that the $S^{A}$ s assign to various members of $\mathcal{A}$. Henceforth, I'll use ' $V^{A}$ ' in place of ' $V_{C}^{A}$ '.

So each of your possible future selves assigns a c-expected value to each of your possible future actions. You must somehow aggregate these individual c-expected values to arrive at an overall opinion that guides your actions. I will assume that your overall opinion takes the form of an expected value function $E$ defined over members of $\mathcal{A}$, together with the maxim that you should choose the $A$ that maximizes $E$. Decision theory, then, can be expressed as a social-welfare functional that takes as input the $\operatorname{set}\left\{V^{A}: A \in \mathcal{A}\right\}$ and yields as output an expected value function $E$ defined over members of $\mathcal{A}$. The choice between e-expected value, c-expected value, and b-expected value is a matter of choosing the right social-welfare functional.

The e-expected value of an action $A$ is determined, to put it metaphorically, by the degree to which $S^{A}$ thinks of himself or herself as a 
suitable candidate for office. In more precise mathematical terms, ${ }^{9}$

$$
V_{E}(A)=V^{A}(A) \text {. }
$$

Metaphorically speaking, the c-expected value of $A$ is determined by taking the weighted average of the degree to which each $S^{B}$ thinks $S^{A}$ is a suitable candidate for office. The weightings are determined by the original agent's beliefs about how likely each of the $S^{B}$ s is to be actualized. (Note that each $S^{B}$ is certain that he or she will be actualized. So the weightings cannot be determined by the opinions of the $S^{B}$ s alone.) Mathematically speaking, ${ }^{10}$

$$
V_{C}(A)=\sum_{B \in \mathcal{A}} V^{B}(A) C(B) .
$$

Finally, metaphorically speaking, the b-expected value of $A$ is determined by the degree to which each $S^{A}$ thinks of himself or herself as a better candidate for office than everybody else. $S^{A}$ 's opinion of everyone else is given by $S^{A}$ 's e-expectation of $B_{K}$. Mathematically speaking, ${ }^{11}$

$$
V_{B T}(A)=V^{A}(A)-\sum_{K \in \mathcal{K}} C^{A}(K) B_{K}
$$

9. Equation (9) should be clear when one compares equations (2) and (7). (2) is simply the special case of (7) where $A=B$.

10. Proof of (10):

Recall (4).

$$
V_{C}(A)=\sum_{K \in \mathcal{K}} C(K) V(A \& K)
$$

The members of $\mathcal{A}$ form a partition, so that $C(K)$ can be rewritten as $\sum_{B \in \mathcal{A}} C(K \mid B) C(B)$. Thus,

$$
V_{C}(A)=\sum_{K \in \mathcal{K}} \sum_{B \in \mathcal{A}} C(K \mid B) C(B) V(A \& K) .
$$

Using the commutative properties of addition and multiplication,

$$
V_{C}(A)=\sum_{B \in \mathcal{K}} \sum_{K \in \mathcal{K}} C(K \mid B) V(A \& K) C(B) .
$$

By (7), $\sum_{K \in \mathcal{K}} C(K \mid B) V(A \& K)$ can be rewritten as $V^{B}(A)$. Thus,

$$
V_{C}(A)=\sum_{B \in \mathcal{A}} V^{B}(A) C(B)
$$

11. Proof of (11):

Recall (5).

$$
V_{B T}(A)=\sum_{K \in \mathcal{K}} C(K \mid A)\left(V(K \& A)-B_{K}\right)
$$


On Wedgwood's way of setting benchmarks, we can rewrite (11) as follows, where $\# \mathcal{A}$ is the number of actions in $\mathcal{A}$ (keeping in mind that $\mathcal{A}$ is chosen so as to exclude all insane actions from consideration).${ }^{12}$

$$
V_{B T}(A)=V^{A}(A)-\frac{\sum_{B \in \mathcal{A}} V^{A}(B)}{\# \mathcal{A}}
$$

So decision theories are in a sense voting procedures, in which both the voters and the candidates for office are your future selves. If we can tell which voting procedure is best, we will be able to ascertain which decision theory is best.

\section{No Adequate Decision Theory}

Advocates of the judgment aggregation approach to voting, in the tradition of Arrow 1951, hold that adequate voting procedures must satisfy certain formal principles. For instance, if a voting procedure is aimed at reaching a democratic decision based on the opinions of various citizens,

We can multiply through the $C(K \mid A)$ factor in each term and divide the sum into two smaller sums as follows.

$$
V_{B T}(A)=\sum_{K \in \mathcal{K}} C(K \mid A) V(K \& A)-\sum_{K \in \mathcal{K}} C(K \mid A) B_{K}
$$

Using (7) to rewrite the first sum, and the definition of $C^{A}$ as $C(\cdot \mid A)$ to rewrite the second,

$$
V_{B T}(A)=V^{A}(A)-\sum_{K \in \mathcal{K}} C^{A}(K) B_{K} .
$$

12. Proof:

According Wedgwood's method of setting benchmarks,

$$
B_{K}=\frac{\sum_{B \in \mathcal{A}} V(K \& B)}{\# \mathcal{A}} .
$$

Thus, for any $A \in \mathcal{A}$ and $K \in \mathcal{K}$,

$$
C^{A}(K) B_{K}=\frac{\sum_{B \in \mathcal{A}} C^{A}(K) V(K \& B)}{\# \mathcal{A}} .
$$

By definition of $V^{A}$,

$$
C^{A}(K) B_{K}=\frac{\sum_{B \in \mathcal{A}} V^{A}(B)}{\# \mathcal{A}} .
$$

And so by (11), using Wedgwood's method of setting benchmarks,

$$
V_{B T}(A)=V^{A}(A)-\frac{\sum_{B \in \mathcal{A}} V^{A}(B)}{\# \mathcal{A}} .
$$


then it must not appoint anyone dictator-there must be no individual whose opinion determines the group decision no matter what.

Likewise, the "voting" procedures used to reach an expected value function $E$ based on the $V^{A}$ s should satisfy certain formal principles. I claim that any adequate decision theory should satisfy the following two principles.

P For any actions $A_{1}$ and $A_{2}$ in $\mathcal{A}$, if for all $B \in \mathcal{A}, V^{B}\left(A_{1}\right) \geq V^{B}\left(A_{2}\right)$ and there is some $B \in \mathcal{A}$ such that $V^{B}\left(A_{1}\right)>V^{B}\left(A_{2}\right)$, then $E\left(A_{1}\right)>E\left(A_{2}\right)$.

S For any decision situations $D=\langle\mathcal{A}, \mathcal{K}, C\rangle$ and $D^{\prime}=\left\langle\mathcal{A}, \mathcal{K}^{\prime}, C^{\prime}\right\rangle$ involving the same set of actions $\mathcal{A}$, if $\mathcal{A}$ contains a pair of actions $A_{1}$ and $A_{2}$ such that $V^{A_{1}}=V^{\prime A_{1}}$ and $V^{A_{2}}=V^{\prime A_{2}}$, then where $E(A)$ is the expected value assigned to $A$ in $D$ and $E^{\prime}(A)$ is the expected value assigned to $A$ in $D^{\prime}$, $E\left(A_{1}\right)>E\left(A_{2}\right)$ iff $E^{\prime}\left(A_{1}\right)>E^{\prime}\left(A_{2}\right)$.

Note that these principles are purely ordinal: they constrain the ordering that $E$ imposes on $\mathcal{A}$ based on the orderings that the $V^{A}$ s impose on $\mathcal{A}$, but they say nothing about the distances between the values assigned to members of $\mathcal{A}$ by either $E$ or the $V^{A}$ s. I will say a bit more to explain and motivate these two principles in turn.

$\mathbf{P}$, a strong Pareto principle, says that if all of the agent's possible future selves like $A_{1}$ at least as well as $A_{2}$, and some of the agent's possible future selves prefer $A_{1}$ to $A_{2}$, then the agent should prefer $A_{1}$ to $A_{2}$. In other words, if you're certain you'll value $A_{1}$ at least as highly as $A_{2}$ no matter which action you perform, and you think you may value $A_{1}$ more highly than $A_{2}$, then $A_{1}$ is strictly better than $A_{2}$.

The Smoking Lesion gets its intuitive appeal from P. P entails that whenever $A_{1}$ weakly dominates $A_{2}$-that is, whenever for every $K \in \mathcal{K}$, $V\left(A_{1} \& K\right) \geq V\left(A_{2} \& K\right)$ and there is some $K \in \mathcal{K}$ such that $C(K)>0$ and $V\left(A_{1} \& K\right)>V\left(A_{2} \& K\right)$-then $E\left(A_{1}\right)>E\left(A_{2}\right) .{ }^{13}$ Smoking dominates

13. Proof: Assume $\mathbf{P}$ holds and $A_{1}$ weakly dominates $A_{2}$. It will suffice to show that $E\left(A_{1}\right)>E\left(A_{2}\right)$. For every $B \in \mathcal{A}$,

$$
V^{B}\left(A_{1}\right)=\sum_{K \in \mathcal{K}} V\left(K \& A_{1}\right) C^{B}(K)
$$

and

$$
V^{B}\left(A_{2}\right)=\sum_{K \in \mathcal{K}} V\left(K \& A_{2}\right) C^{B}(K) .
$$

If $A_{1}$ weakly dominates $A_{2}$, then every term in the second sum can be paired with a term of equal or greater value in the first sum. So for every $B \in \mathcal{A}, V^{B}\left(A_{1}\right) \geq V^{B}\left(A_{2}\right)$. Furthermore, if $A_{1}$ weakly dominates $A_{2}$, there is some $K \in \mathcal{K}$ such that $V\left(A_{1} \& K\right)>V\left(A_{2} \& K\right)$ and $C(K)>0$. Since the members of $\mathcal{A}$ form a partition, and since I've assumed that the agent is a conditionalizer, there must be some $B \in \mathcal{A}$ such that, for the $K$ just mentioned, 
(and hence weakly dominates) not smoking. This is exactly why smoking seems like the right answer: it will make Susan better off no matter what!

S might be deemed a "self-sovereignty" condition. It states that whether $E\left(A_{1}\right)$ is greater than $E\left(A_{2}\right)$ should depend solely on the opinions of $S^{A_{1}}$ and $S^{A_{2}}$ —in other words, only on what the agent will believe and desire after performing $A_{1}$ and what the agent will believe and desire after performing $A_{2}$. This idea is intuitively appealing: the point of choosing for your future self is to end up with a future self who is in some sense happy to exist. So whether you should choose one future self $S^{A_{1}}$ over another future self $S^{A_{2}}$ is up to what $S^{A_{1}}$ and $S^{A_{2}}$ would think if one of them came to exist. It is not about what other future selves would think of $S^{A_{1}}$ and $S^{A_{2}}$ if neither $S^{A_{1}}$ nor $S^{A_{2}}$ came to exist. Nor (crucially) is it about how likely $S^{A_{1}}$ and $S^{A_{2}}$ are to come into existence, by the agent's current lights.

The Psychopath Button gets its intuitive appeal from $\mathbf{S}$. It seems that whether pressing is better than not pressing depends only on what Paul can reasonably expect to happen after pressing and on what Paul can reasonably expect to happen after not pressing. Whether Paul is likely to press is irrelevant.

So $\mathbf{P}$ and $\mathbf{S}$ are both intuitively appealing as constraints on rational decision making. Both of them play important roles in motivating the counterexamples above. Unfortunately, neither EDT, CDT, nor BT satisfies both of them. In fact, it is impossible for any completely general decision theory to satisfy both $\mathbf{P}$ and $\mathbf{S}$.

The incompatibility can be proved by constructing a pair of decision situations that cannot individually satisfy $\mathbf{P}$ while jointly satisfying S. Rather than fully specifying one such pair of situations, I will give a schematic general recipe for constructing pairs of decision situations that raise the problem. Notice that the schematic recipe requires decision situations in which the agent must choose among three or more distinct actions. As we'll see, this is no accident.

The construction is as follows. Let $D=\langle\mathcal{A}, \mathcal{K}, C\rangle$ and $D^{\prime}=$ $\left\langle\mathcal{A}, \mathcal{K}^{\prime}, C^{\prime}\right\rangle$ be two decision situations involving the same set of actions $\mathcal{A}$. Let $\mathcal{A}$ contain two distinct actions $A_{1}$ and $A_{2}$, and let $\mathcal{F}$ be a nonempty subset of $\mathcal{A}$ containing all and only actions distinct from both $A_{1}$ and $A_{2}$. Where $V^{B}(A)$ is the value that $S^{B}$ assigns to $A$ in $D$ and $V^{\prime B}(A)$ is the

$\overline{C^{B}(K)}>0$. Hence, there is some $B \in \mathcal{A}$ such that $V\left(K \& A_{1}\right) C^{B}(K)>V\left(K \& A_{1}\right) C^{B}(K)$. For this $B, V^{B}\left(A_{1}\right)>V^{B}\left(A_{2}\right)$. By the Pareto Principle, $E\left(A_{1}\right)>E\left(A_{2}\right)$. 
value that $S^{B}$ assigns to $A$ in $D^{\prime}$, let $V^{A_{1}}=V^{A_{2}}=V^{\prime A_{1}}=V^{\prime A_{2}}$. Finally, for all $F \in \mathcal{F}$, let $V^{F}\left(A_{1}\right)>V^{F}\left(A_{2}\right)$, and let $V^{\prime F}\left(A_{1}\right)<V^{\prime F}\left(A_{2}\right)$.

For all $B \in \mathcal{A}, V^{B}\left(A_{1}\right) \geq V^{B}\left(A_{2}\right)$. Furthermore, since I've stipulated that $\mathcal{F}$ is nonempty, there is some $B \in \mathcal{A}$ such that $V^{B}\left(A_{1}\right)>V^{B}\left(A_{2}\right)$. Therefore, $\mathbf{P}$ requires that $E\left(A_{1}\right)>E\left(A_{2}\right)$. Likewise, for all $B \in \mathcal{A}$, $V^{\prime B}\left(A_{2}\right) \geq V^{\prime B}\left(A_{1}\right)$, and there is some $B \in \mathcal{A}$ such that $V^{\prime B}\left(A_{2}\right)>$ $V^{\prime B}\left(A_{1}\right)$. Therefore, $\mathbf{P}$ requires that $E^{\prime}\left(A_{2}\right)>E^{\prime}\left(A_{1}\right)$. But $V^{A_{1}}=V^{\prime A_{1}}$ and $V^{A_{2}}=V^{\prime A_{2}}$, so $\mathbf{S}$ requires that $E(A)>E(B)$ iff $E^{\prime}(A)>E^{\prime}(B)$. In this schematic example, $\mathbf{P}$ 's requirements contradict $S$ 's requirements, so the two principles are incompatible. ${ }^{14}$

Although no decision theory can satisfy both rules at once, each of the three decision theories I've discussed satisfies one of them. EDT and Wedgwood's version of BT satisfy $\mathbf{S}$ but violate $\mathbf{P}$, while CDT satisfies $\mathbf{P}$ but violates $\mathbf{S}$.

The fact that EDT and BT satisfy $\mathbf{S}$ can be read off equations (9) and (11). $V_{E}(A)=V^{A}(A)$, and $V_{B T}(A)$ is a function of $V^{A}(A)$ and $\# \mathcal{A}$. So where $D=\langle\mathcal{A}, \mathcal{K}, C\rangle$ and $D^{\prime}=\left\langle\mathcal{A}, \mathcal{K}^{\prime}, C^{\prime}\right\rangle$ are decision situations such that $V^{A}(A)=V^{\prime A}(A)$, it must be true that both $V_{E}(A)=V_{E}^{\prime}(A)$ and $V_{B T}(A)=V_{B T}^{\prime}(A)$. Likewise, where $D=\langle\mathcal{A}, \mathcal{K}, C\rangle$ and $D^{\prime}=\left\langle\mathcal{A}, \mathcal{K}^{\prime}, C^{\prime}\right\rangle$ are decision situations such that $V^{B}(B)=V^{\prime B}(B)$, it must be true that both $V_{E}(B)=V_{E}^{\prime}(B)$ and $V_{B T}(B)=V_{B T}^{\prime}(B)$. Thus, where $D=\langle\mathcal{A}, \mathcal{K}, C\rangle$ and $D^{\prime}=\left\langle\mathcal{A}, \mathcal{K}^{\prime}, C^{\prime}\right\rangle$ are decision situations such that $V^{A}(A)=V^{\prime A}(A)$ and $V^{B}(B)=V^{\prime B}(B)$, it must be true that $V_{E}(A)>V_{E}(B)$ iff $V_{E}^{\prime}(A)=V_{E}^{\prime}(B)$, and that $V_{B T}(A)>V_{B T}(B)$ iff $V_{B T}^{\prime}(A)=V_{B T}^{\prime}(B)$.

The fact that EDT and BT violate $\mathbf{P}$ follows from the fact that they sometimes recommend weakly dominated options. EDT recommends the weakly dominated option in The Smoking Lesion, while BT recommends the weakly dominated option in The Three-Option Smoking Lesion.

CDT satisfies $\mathbf{P}$. Fleming 1952 shows that $\mathbf{P}$ can be combined with a plausible independence principle so that the two principles are jointly satisfied if and only if (in our present parlance) $E(A)$ is a weighted average of the $V^{A} \mathbf{s} . V_{C}$ is a weighted average of the $V^{A} \mathbf{s}$, a fact that is clear from equation (10).

But CDT violates $\mathbf{S}$ even in cases where there are only two optionsa fact that can be illustrated using a variant on The Psychopath Button.

14. This problem is closely akin to the Liberal Paradox (Sen 1982). In the Liberal Paradox, a weak Pareto principle entailed by $\mathbf{P}$ is shown to be incompatible with a liberal principle that assigns to each voter a pair of options $A$ and $B$ such that society prefers $A$ to $B$ if and only if the corresponding voter does. 
Suppose $\mathcal{A}$ and $\mathcal{K}$ are specified as in the original version of the example, but $C$ is given by the following alternative matrix (where the overbar denotes a repeating decimal).

\begin{tabular}{|l|l|l|}
\hline & $P$ & $\neg P$ \\
\hline$B$ & .81 & .09 \\
\hline$\neg B$ & $.00 \overline{1}$ & $.09 \overline{8}$ \\
\hline
\end{tabular}

By (4),

$$
\begin{aligned}
V_{C}(B) & =C(P) V(P \& B)+C(\neg P) V(\neg P \& B) \\
& =(.8 \overline{1})(-100)+(.1 \overline{8})(20) \\
& \approx-77 \\
V_{C}(\neg B) & =C(P) V(P \& \neg B)+C(\neg P) V(\neg P \& \neg B) \\
& =(.8 \overline{1})(0)+(.1 \overline{8})(0) . \\
& =0
\end{aligned}
$$

Unlike in the original version of the example, $V_{C}(B)<V_{C}(\neg B)$.

But notice that Paul's credences conditional on $P$ and conditional on $\neg P$ are completely unchanged, and the dependency hypotheses are completely unchanged as well. So $V^{B}$ and $V^{\neg}$ are exactly the same as in the original case. The moral: Whether $P$ 's c-expected value is greater than that of $\neg P$ depends not just on the $V^{A} \mathrm{~s}$, but on the weightings assigned to them by the original agent.

Wedgwood's version of BT has one noteworthy advantage over CDT and EDT. EDT violates $\mathbf{P}$ even in decision situations with just two options. (This is what happens in The Smoking Lesion.) And CDT violates $\mathbf{S}$ even in decision situations with just two options. (This is what happens in the two versions of The Psychopath Button.) But in decision situations where $\mathcal{A}$ contains only two actions, BT (taken together with Wedgwood's method of setting benchmarks) satisfies both $\mathbf{P}$ and $\mathbf{S}$.

We know that BT satisfies $\mathbf{S}$ in two-option cases because it satisfies $\mathbf{S}$ in all cases. To show that BT satisfies $\mathbf{P}$ in two-option cases, we simply assume that the antecedent of $\mathbf{P}$ is satisfied and prove the consequent. In other words, where $\mathcal{A}=\left\{A_{1}, A_{2}\right\}$, we assume that either $V^{A_{1}}\left(A_{1}\right) \geq V^{A_{1}}\left(A_{2}\right)$ and $V^{A_{2}}\left(A_{1}\right)>V^{A_{2}}\left(A_{2}\right)$, or $V^{A_{2}}\left(A_{1}\right) \geq V^{A_{2}}\left(A_{2}\right)$ and 
$V^{A_{1}}\left(A_{2}\right)>V^{A_{1}}\left(A_{2}\right)$. We can then prove that $V_{B T}\left(A_{1}\right)>V_{B T}\left(A_{2}\right)$. By $(11)$,

$$
\begin{aligned}
V_{B T}\left(A_{1}\right) & =V^{A_{1}}\left(A_{1}\right)-\frac{V^{A_{1}}\left(A_{1}\right)+V^{A_{1}}\left(A_{2}\right)}{2} \\
& =\frac{V^{A_{1}}\left(A_{1}\right)-V^{A_{1}}\left(A_{2}\right)}{2}
\end{aligned}
$$

and

$$
\begin{aligned}
V_{B T}\left(A_{2}\right) & =V^{A_{2}}\left(A_{2}\right)-\frac{V^{A_{2}}\left(A_{1}\right)+V^{A_{2}}\left(A_{2}\right)}{2} \\
& =\frac{V^{A_{2}}\left(A_{2}\right)-V^{A_{2}}\left(A_{1}\right)}{2} .
\end{aligned}
$$

If $V^{A_{1}}\left(A_{1}\right) \geq V^{A_{1}}\left(A_{2}\right)$ and $V^{A_{2}}\left(A_{1}\right)>V^{A_{2}}\left(A_{2}\right)$, then $V_{B T}\left(A_{1}\right) \geq 0$ and $V_{B T}\left(A_{2}\right)<0$. If $V^{A_{2}}\left(A_{1}\right) \geq V^{A_{2}}\left(A_{2}\right)$ and $V^{A_{1}}\left(A_{2}\right)>V^{A_{1}}\left(A_{2}\right)$, then $V_{B T}\left(A_{1}\right)>0$ and $V_{B T}\left(A_{2}\right) \leq 0$. In either case, $V_{B T}\left(A_{1}\right)>V_{B T}\left(A_{2}\right)$-the consequent of $\mathbf{P}$ is satisfied.

BT satisfies both $\mathbf{P}$ and $\mathbf{S}$ in all two-option cases. This is what made it look so attractive in The Smoking Lesion and The Psychopath Button, each of which involved a choice between only two actions. But BT does not satisfy $\mathbf{P}$ and $\mathbf{S}$ in general. No decision rule does.

\section{The Tickle Defense}

No decision rule is perfect. What's a decision-maker to do? One possible solution-an analogue of the position advocated by List 2003 in response to certain voting paradoxes-is to avoid situations where plausible decision rules conflict. Philosophers wishing to pursue this strategy have a ready-made avoidance strategy at hand: the tickle defense.

According to the tickle defense, decision situations like The Smoking Lesion, The Psychopath Button, and The Three-Option Smoking Lesion are poorly described and should be ruled impossible at the outset. Any agent (or at least, any suitable agent) in any decision situation (or at least, any suitable decision situation) has some piece of knowledge that renders his or her actions evidentially independent of the dependency hypotheses. In other words, for any credence function $C$ that could represent the beliefs of a suitable agent in a suitable decision situation, $C(K)=C(K \mid A)$ for each $A \in \mathcal{A}$ and $K \in \mathcal{K}$. Therefore, whenever a suitable agent faces a suitable decision situation, EDT, CDT, and BT will give exactly the same advice.

So says the tickle defense, outlined abstractly. But what ensures that for suitable agents in suitable decision situations, the As are independent 
of the Ks? I'll explain the answer by first showing why The Smoking Lesion seems unsuitable and then generalizing the point to a class of related examples.

In The Smoking Lesion, Susan does not know whether she will get cancer. But if EDT and CDT are to conflict, Susan's smoking must provide her with evidence that she will get cancer. The evidential link between smoking and cancer is explained by the hypothesis that the two events have a common cause-the lesion. But how (according to Susan's beliefs) might the lesion cause smoking?

Perhaps Susan thinks the lesion makes victims feel some sort of desire or "tickle" that can only be assuaged by tobacco. But surely Susan already knows by introspection whether she has the tickle or not. If she has the tickle, then she knows that she has (or is likely to have) the lesion, regardless of whether she smokes. If she lacks the tickle, then she knows that she lacks (or is likely to lack) the lesion, again regardless of whether she smokes. Once she knows about the presence or absence of the tickle, all her evidence about the presence or absence of the lesion is in. In light of what Susan knows, $S$ is evidentially independent of $R$.

On the other hand, perhaps Susan thinks the lesion causes smoking not by influencing agents' desires, but by influencing their beliefs. Perhaps (on Susan's view of things) people with the lesion tend to believe smoking is harmless, while people without the lesion tend to believe smoking causes lung cancer. But surely Susan knows what she believes. (In fact, it's stipulated that she thinks smoking is harmless.) Whether or not Susan ends up smoking, the evidence about the lesion is in. Once again, in light of what Susan knows, $S$ is evidentially independent of $R$.

So on either way of filling in the example, the matrix I've used to describe $C$ is wrong. It cannot be that $C(R \mid S)>C(R \mid \neg S)$. Proponents of the tickle defense claim there is no adequate way of filling in the example that will secure $R$ 's evidential dependence on $S$. This is because-to follow the version of the tickle defense proposed by Eells 1982-a suitable agent must be rational and self-aware enough to use decision theory, and must believe that he or she is.

Being rational, says Eells, is a matter of having beliefs (that is, a credence function $C$ ) and desires (that is, a value function $V$ ) that are jointly causally sufficient to determine one's actions. Being self-aware is a matter of knowing what one's beliefs and desires are. And believing that one is rational is a matter of believing that one's actions are causally determined by one's beliefs and desires (even if one is not certain how the causal determination works, so that one is not certain what one will do). 
If Susan believes she is rational, then she believes that the lesion can affect her choice to smoke only by affecting her beliefs and desires. If she is self-aware, however, then she knows what her beliefs and desires are. Given this information, information about whether she smokes cannot tell her anything further about whether she has the lesion. (Eells points out that all this is perfectly compatible with Susan's treating other people's smoking or not smoking as evidence about whether they have the lesion. Susan need not believe that other people are rational and need not be aware of what they believe and desire.)

The Smoking Lesion, The Psychopath Button, and The Three-Option Smoking Lesion all explain the evidential dependency between the agent's actions and the dependency hypotheses by appealing to some clinical condition that serves as a common cause of both actions and certain dependency hypotheses' being true. But when it comes to actions and the truth of true dependency hypotheses, the presence of a common cause does not entail evidential dependence. A suitable agent will always know his or her beliefs and desires, and so will possess all the evidence about dependency hypotheses that might have been furnished by his or her actions. This is why a suitable agent possesses knowledge that renders his or her actions evidentially independent of the dependency hypotheses.

What does all this have to do with voting? Whenever $C(K)=$ $C(K \mid A)$ for each $A \in \mathcal{A}$ and $K \in \mathcal{K}$, it follows that $V^{F}(A)=V^{G}(A)$ for all $A, F, G \in \mathcal{A}$. In other words, for any $A \in \mathcal{A}$, the agent's future selves are in exact agreement as to $A$ 's c-expected value. If the tickle defense is right, then every decision situation involves taking a unanimous vote among the agent's actions. If unanimity is guaranteed at the outset, then no voting paradoxes (or decision-theoretic paradoxes) can arise.

Whether the tickle defense rules out all cases of conflict between EDT, CDT, and BT is somewhat controversial. Perhaps agents can be reasonable enough to look to decision theory for advice without being as rational and self-aware as Eells requires. (See Horwich 1987, Eells 1984, and Sobel 1994 for further discussion.) But whether or not Eells was right to claim that all suitable agents have information that renders their actions evidentially irrelevant to the dependency hypotheses, it seems clear that a great many suitable agents will have information that renders their actions evidentially irrelevant to the dependency hypotheses. To the extent that situations like The SmokingLesion, The Psychopath Button, and The Three-Option Smoking Lesion are rare, the task of deciding among various possible decision theories is rendered much less urgent. 


\section{Conclusion}

EDT fails in medical Newcomb problems like The Smoking Lesion, CDT fails in cases like The Psychopath Button, and BT fails in cases like The Three Option Smoking Lesion.

We're unlikely to find any decision rule that succeeds where CDT, EDT, and BT have failed. Decision-theoretic rules can be recast as voting rules, and just as there are sets of reasonable desiderata that cannot be jointly satisfied by any voting rule, there are sets of reasonable desiderata that cannot be jointly satisfied by any decision rule. In particular, good decision rules should satisfy—but cannot satisfy—both a Pareto constraint and a "self-sovereignty" principle.

Luckily, few agents need decision rules that perform well in every conceptually possible situation. It is enough to have a decision rule that performs well in the overwhelming majority of situations one is likely to encounter. Thanks to the tickle defense, EDT, CDT, and BT agree in all but a handful of unlikely situations. Therefore, in most situations, all three rules are equally acceptable. This happy situation in decision theory is the formal analogue of a much rarer situation in judgment aggregationnamely, total social consensus.

\section{References}

Arrow, Kenneth. 1951. Social Choice and Individual Values. New York: Wiley. Bostrom, Nick. 2001. "The Meta-Newcomb Problem." Analysis 61: 309-10.

Eells, Ellery. 1982. Rational Decision and Causality. Cambridge: Cambridge University Press.

—. 1984. "Newcomb's Many Solutions." Theory and Decision 16: 59-105.

- 1985. "A Reply to Jackson and Pargetter." In Paradoxes of Rationality and Cooperation, ed. Richmond Campbell and Lanning Sowden, 219-23. Vancouver: University of British Columbia Press.

Egan, Andy. 2007. "Some Counterexamples to Causal Decision Theory." Philosophical Review 116: 93-114.

Fleming, Marcus. 1952. "A Cardinal Concept of Welfare." Quarterly Journal of Economics 66: 366-84.

Gibbard, Allan, and William Harper. 1978. "Counterfactuals and Two Kinds of Expected Utility." In Foundations and Applications of Decision Theory, ed. C. A. Hooker, J. J. Leach, and E. F. McClennen, 125-62. Dordrecht, Holland: Reidel.

Horwich, Paul. 1987. Asymmetries in Time. Cambridge, MA: MIT Press.

Jeffrey, Richard. 1983. The Logic of Decision, 2nd ed. Chicago: University of Chicago Press. 
Joyce, James. 1999. The Foundations of Causal Decision Theory. Cambridge: Cambridge University Press.

Lewis, David. 1981. "Causal Decision Theory." Australasian Journal of Philosophy 59: 5-30.

List, Christian. 2003. "A Possibility Theorem on Decisions over Multiple Propositions." Mathematical Social Sciences 45: 1-13.

Loomes, Graham, and Robert Sugden. 1982. "Regret Theory: An Alternative Theory of Rational Choice under Uncertainty.” Economic Theory 92: 805-24.

Sen, Amartya. 1982. Choice, Welfare, and Measurement. Oxford: Basil Blackwell.

Sobel, Jordan Howard. 1994. Taking Chances: Essays on Rational Choice. Cambridge: Cambridge University Press.

Wedgwood, Ralph. 2009. "Gandalf's Solution to the Newcomb Problem." http:/ / users.ox.ac.uk/ mert1230/gandalf.a4.pdf (accessed July 21, 2009). 